\title{
铝/钢连续驱动摩擦焊焊接扭矩和能量输入特征
}

\author{
张昌青 $^{1}$ 刘雄波 ${ }^{1}$ 吕广明 ${ }^{1}$ 苏执元 $^{2}$ 李京龙 $^{3}$ \\ (1. 兰州理工大学材料科学与工程学院 兰州 730050; \\ 2. 兰州理工大学机电工程学院 兰州 730050 ;
}

3. 西北工业大学材料学院 西安 710072)

\begin{abstract}
摘要: 基于摩擦扭矩是连续驱动摩擦焊焊接界面摩擦阻力做功的综合体现, 采用主电动机定子电压电流法(Voltage and current of main motor, VCMM), 获得了 1050 纯铝和 E235 低碳钢连续驱动摩擦焊过程焊接界面的摩擦扭矩, 分析转速、顶锻压力对 接头摩擦扭矩和不同焊接阶段能量输入的影响。结果表明: 初始摩擦阶段是接头热量的积累阶段, 以粘着摩擦产热为主; 转 速较低时, 接头摩擦扭矩曲线只存在一个峰值或前后峰值特征不明显, 此时接头发生失稳摩擦, 转速升高, 扭矩降低, 初始 摩擦阶段、准稳态阶段和焊接全过程能量输入都增加, 但由于顶锻过程摩擦加热功率的降低使得顶锻阶段能量输入缓慢减小; 后峰值扭矩和顶锻阶段能量输入与顶锻压力的变化呈正相关。
\end{abstract}

关键词: 连续驱动摩擦焊; 转速; 顶锻压力; 扭矩; 能量输入

中图分类号: TG456

\section{Friction Torque and Heat Input Characteristics During Continuous Drive Friction Welding of Aluminum to Steel}

\author{
ZHANG Changqing $^{1}$ LIU Xiongbo ${ }^{1}$ LÜ Guangming ${ }^{1} \quad$ RUI Zhiyuan ${ }^{2}$ LI Jinglong ${ }^{3}$ \\ (1. School of Materials Science and Engineering, Lanzhou University of Technology, Lanzhou 730050; \\ 2. School of Mechanical and Electrical Engineering, Lanzhou University of Technology, Lanzhou 730050; \\ 3. School of Materials Science and Engineering, Northwestern Polytechnical University, Xi’an 710072)
}

\begin{abstract}
Friction torque is a comprehensive manifestation of friction resistance during the continuous drive friction welding. By monitoring the real-time voltage and current of main motor stator during the continuous drive friction welding of 1050 pure aluminum to E235 steel, evolutions of friction torque on the joint interface were obtained and the effects of rotation speed and forging pressure on the friction torque and heat input of each welding stage are analyzed. The results show that the initial friction stage is the process of heat input accumulation, where "stick" friction heat generation is predominant. When rotation speed is low, friction torque curve only has a peak or the initial and final peak features are not obvious, the whole friction process is unstable. With the increasing of rotation speed, friction torque increases, the heat input of initial friction stage, the quasi-steady state stage and the whole process is increased, but heat input in forging stage is slowly reduced due to the decrease of the friction heating power during the forging stage. Final peak torque and heat input in forging stage are positively correlated with the change of forging pressure.
\end{abstract}

Key words: continuous drive friction welding; friction torque; rotation speed; forging pressure; heat input

\section{0 前言}

随着现代制造业的飞速发展, 连续驱动摩擦焊作 为一种高质量、高效率、环保的固相焊方法 ${ }^{[1-2]}$, 在异 种材料的先进焊接制造中显示出巨大的潜力 ${ }^{[3-4]}$ 。连续 驱动摩擦焊是以焊接界面摩擦阻力做功为热源, 而 接头界面摩擦阻力做功的综合体现形式是摩擦扭矩

20170524 收到初稿, 20170724 收到修改稿
的变化。摩擦扭矩不仅可用来表征接头界面的摩擦 特性, 同时也与摩擦界面金属的塑性变形和流动、 接头能量输入及温度场分布紧密相关 ${ }^{[5-7]}$, 这些都会 直接影响焊缝元素扩散和接头组织的再结晶、相变 行为, 最终关系到接头的力学性能, 所以对接头摩 擦扭矩的分析和研究是十分必要的。

KIMURA 等 ${ }^{[8]}$ 认为摩擦扭矩的多峰值现象反映 了工件接触表面状态的不稳定性, 近几年他还给出 了摩擦焊接头界面热源的演变过程, 并采用统计回 归的方法得出了不同焊接参数下接头界面发生粘着 
温度的唯象模型 ${ }^{[9]}$, 但该模型的物理意义尚需要进 一步探讨以获普适性。LI 等 ${ }^{[10]}$ 结合摩擦扭矩的变化 对 20 中碳钢连续驱动摩擦焊过程中的初始摩擦阶 段作了探讨, 研究发现, 当摩擦压力较小时扭矩曲 线出现了明显的平台特征, 且整个初始摩擦阶段接 头界面温度持续上升。通常焊接过程中接头摩擦扭 矩变化幅度大且频率快, 往往需要采集系统具有良 好的静、动态性能, 主电动机定子电压电流法 (Voltage and current of main motor, VCMM)对焊接 过程中扭矩的动态变化有较好的适应性 ${ }^{[11]}$, 使用较 广泛。 $\mathrm{LUO}^{[12]}$ 在 VCMM 法的基础上对摩擦加热功 率进行浮动求差, 测得了直径 $27 \mathrm{~mm}$ 的 $65 \mathrm{Mn}$ 连续 驱动摩擦焊接头焊接过程中摩擦扭矩的变化曲线, 并对该曲线进行了回归分析, 建立了初始摩擦阶段 到稳态阶段的摩擦扭矩数理模型。

本文采用 VCMM 法获得了 1050 纯铝和 E235 低碳钢连续驱动摩擦焊过程焊接界面的摩擦扭矩, 通过摩擦扭矩对摩擦时间的积分运算分析了不同转 速、顶锻压力下接头在初始摩擦阶段、准稳态阶段 和顶锻阶段的能量输入特征, 为铝/钢异种金属及其 他异种金属的高效率、高强度和可靠性摩擦焊接提 供一些理论依据。

\section{1 试验与原理}

\section{1 试验}

试验材料选用 1050 纯铝棒和 E235 低碳钢棒。 1050 纯铝棒尺寸为 $\phi 25 \mathrm{~mm} \times 100 \mathrm{~mm}$; E235 低碳钢 棒总长 $80 \mathrm{~mm}$, 夹持端为 $\phi 25 \mathrm{~mm} \times 50 \mathrm{~mm}$, 焊接端 面直径为 $40 \mathrm{~mm}$ 。材料的主要化学成分如表 1 所示, 材料焊前的宏观照片如图 1 所示。本试验所用的设 备为 C320 型连续驱动摩擦焊机。该焊机主电动机 为直流调速电机, 具有抗干扰能力强、可靠性好、 数据采集准确度高等优点。配备的计算机闭环控制 系统 ${ }^{[13-14]}$ 可对焊接过程中的轴向压力和摩擦缩短量 进行实时双闭环控制, 配备的电压电流监测系统可 实时监测焊接过程中主电动机转速以及定子电压和 电流, 采样频率为 $1000 \mathrm{~Hz}$ 。

表 1 母材的主要化学成分(质量分数) \%

\begin{tabular}{ccccccccc}
\hline & $\mathrm{C}$ & $\mathrm{Mn}$ & $\mathrm{Si}$ & $\mathrm{P}$ & $\mathrm{S}$ & $\mathrm{Mg}$ & $\mathrm{Fe}$ & $\mathrm{Al}$ \\
\hline 1050 & - & 0.05 & 0.25 & - & - & 0.05 & 0.40 & 余量 \\
$\mathrm{E} 235$ & 0.18 & 1.4 & 0.3 & 0.045 & 0.045 & - & 余量 & - \\
\hline
\end{tabular}

焊前用丙酮和无水乙醇清洗钢侧端面和铝侧端 面, 将铝棒作为旋转端, 钢棒作为工进端, 由于摩 擦焊中各个工艺参数的选用范围很广, 不同的组合 可得到不同的工艺规范, 本文主要针对转速和顶锻
压力对焊接扭矩和不同阶段能量输入的影响进行讨 论, 具体工艺参数如表 2 所示。

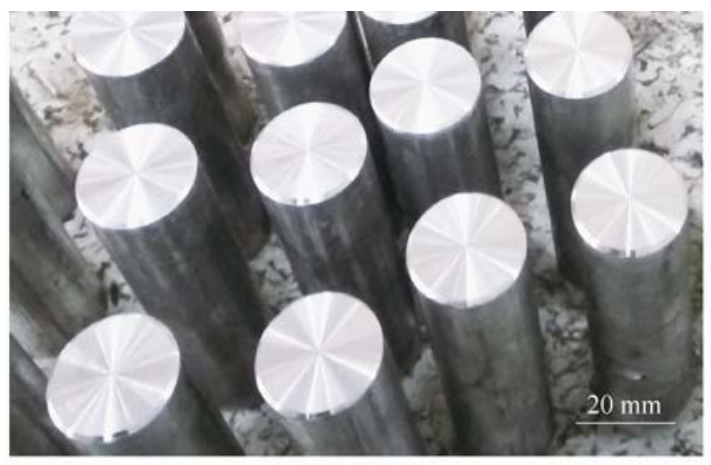

(a) 1050 纯铝

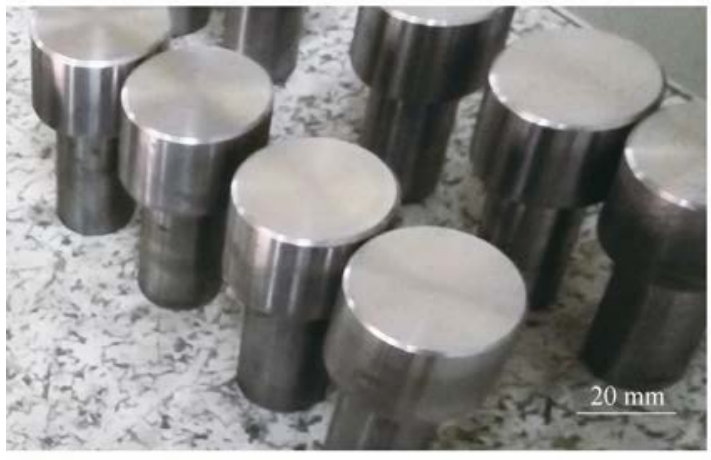

(b) E235 低碳钢

图 1 试验材料宏观照片

表 2 试验所采用的工艺参数

\begin{tabular}{cccc}
\hline 转速/(r/min) & 摩擦压力/MPa & 顶锻压力/MPa & 摩擦缩短量 $/ \mathrm{mm}$ \\
\hline $\begin{array}{c}300,500,800,1500, \\
2000,2500\end{array}$ & 30 & 60 & 5 \\
1500 & 30 & $60,80,100,120$ & 5 \\
\hline
\end{tabular}

\section{2 试验原理}

为保证试验的重复性、可靠性, 每组工艺参 数下开展三次焊接试验, 分别将三次测的电流电 压计算后得到同一工艺参数下的三条扭矩曲线, 选取重复性较好的一条进行对比分析。下面以转速 $1500 \mathrm{r} / \mathrm{min}$ 、摩擦压力 $30 \mathrm{MPa}$ 、顶锻压力 $60 \mathrm{MPa}$ 、 摩擦缩短量 $5 \mathrm{~mm}$ 时的焊接过程为例来说明扭矩曲 线的获得过程。

当主电动机在空载运行时, 输入功率

$$
P_{0}=P_{a}+P_{b}=U_{0} \bullet I_{0}
$$

式中, $P_{a}$ 为空载时主电动机内部的损耗功率; $P_{b}$ 为 空载时传动系统的损耗功率, $U_{0}$ 和 $I_{0}$ 分别为空载运 行时实时监测的电压和电流。

焊接开始后, 主电动机输入功率

$$
P_{1}=P_{a}{ }^{\prime}+P_{b}{ }^{\prime}+P_{f}=U_{1} \cdot I_{1}
$$

式中, $P_{a}{ }^{\prime}$ 为加载时主电动机内部损耗的功率; $P_{b}{ }^{\prime}$ 为 加载时焊机传动系统损耗的功率; $P_{f}$ 为工件界面摩 擦加热功率。 $U_{1}$ 和 $I_{1}$ 分别为焊接开始后实时采集的 
电压和电流。图 2 所示为实时监测的主电动机定子 的电压、电流变化曲线, 可以看出整个焊接过程电 压变化波动较小, 空载时电流波动平稳, 当焊接开 始后(图 2 中 $A$ 点开始)电流变化显著, 出现了前后 两个峰值。

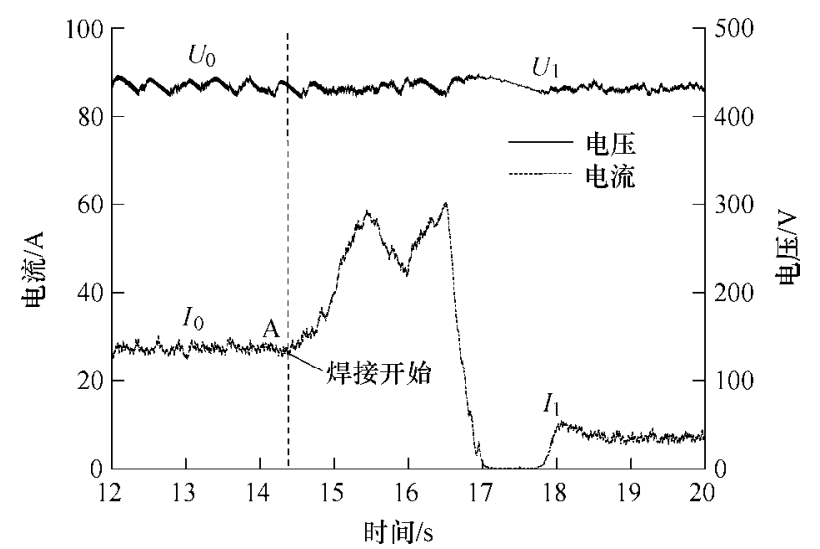

图 2 焊接过程中主电动机定子的电压、电流变化曲线

主电动机的自身损耗和传动系统的损耗在空载 时和焊接时相差并不大, 并且占总能量消耗的比例 很小, 因此可以认为

$$
P_{a}=P_{a}^{\prime} \quad P_{b}=P_{b}{ }^{\prime}
$$

由式(1) (3)可得摩擦功率

$$
P_{f}=P_{1}-P_{0}=U_{1} \bullet I_{1}-U_{0} \bullet I_{0}
$$

根据连续驱动摩擦焊经典产热模型 ${ }^{[4]}$, 摩擦扭 矩 $T$ 和摩擦加热功率 $P_{f}$ 分别为

$$
\begin{gathered}
T=2 \pi \int_{0}^{R} f(r, t) p(r, t) r^{2} \mathrm{~d} r \\
P_{f}=2 \pi \omega \int_{0}^{R} f(r, t) p(r, t) r^{2} \mathrm{~d} r
\end{gathered}
$$

式中, $\omega$ 为角速度; $R$ 为工件半径; $f(r, t)$ 为摩擦因 数; $p(r, t)$ 为轴向压力; $r$ 为摩擦界面某点到轴心的 距离; $t$ 为摩擦时间。

综上, 摩擦扭矩

$$
T=P_{f} / \omega
$$

图 3 为由式(1) (7)推算出的焊接接头摩擦扭 矩变化曲线, 呈现出和主电动机定子电流 $A$ 点以后 几乎相同的变化趋势。

通常认为, 焊接过程中接头界面摩擦扭矩做功 产生的机械能可以全部转化为焊接摩擦热, 所以, 摩擦扭矩曲线和时间轴所围成的面积则反映了输入 到焊接界面的能量。根据得出的扭矩曲线, 不同时 间段接头的能量输入 $H$ 可表示为

$$
H=\omega \int_{t 1}^{t_{2}} T \mathrm{~d} t
$$

然后对不同工艺参数下的焊接扭矩曲线进行比 对分析, 同时讨论接头在接头形成不同阶段的能量
输入情况。

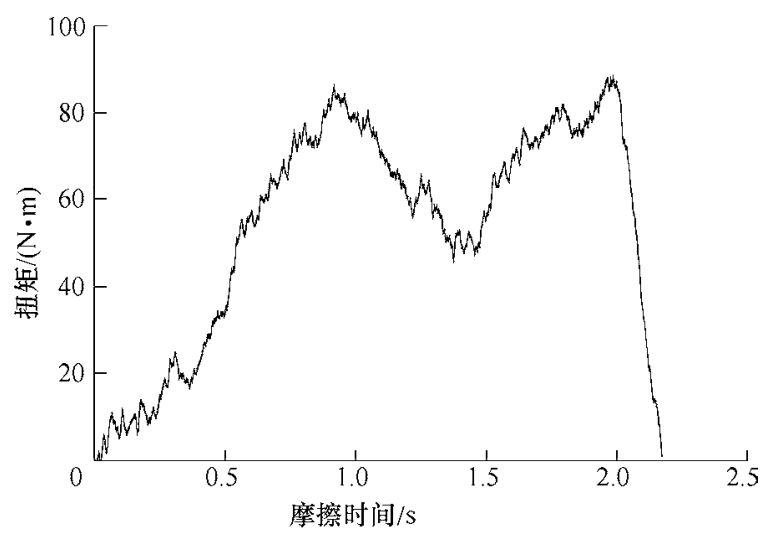

图 3 计算得出的焊接接头摩擦扭矩的变化曲线

\section{2 结果与分析}

\section{1 焊接过程阶段划分}

将 1.2 中得到的摩擦扭矩曲线经 Savitzky-Golay 滤波器滤波后如图 4b 所示, 传统上根据摩擦扭矩的 变化特征可将整个焊接过程分为三个阶段：初始摩 擦阶段(第一阶段)、准稳态阶段(第二阶段)、顶锻阶 段(第三阶段 $)^{[1-2]}$ 。图 $4 \mathrm{a}$ 为焊接过程中接头的结合形 貌。(1)、(2)、(3)对应初始摩擦阶段, 经发展, 对旋 转摩擦焊初始摩擦阶段的认识已经深化到可以细分 为三个阶段, 即 $\mathrm{LI}$ 等 $^{[10]}$ 描述的磨损(Abrasion)阶段、 MAALEKIAN 等 ${ }^{[15]}$ 描述的滑动摩擦(Slide)阶段和 KIMURA 等 ${ }^{[9]}$ 描述的粘着摩擦(Stick)阶段。由图 4b 可以看出铝和钢的连续驱动摩擦焊也具备这种特 征, 随着钢端界面和铝端界面的接触, 焊接过程开 始, 界面的一些微凸体互相磨损、剪切产生摩擦热 对接头起到预热作用, 扭矩升高后停留在一个平台, 这个平台对应的就是滑动摩擦阶段, 之后摩擦扭矩 逐渐上升到初始峰值扭矩, 此时热塑性的铝已扩展 至整个界面; (4)对应准稳态阶段, 由于摩擦扭矩和 摩擦压力的作用, 界面不断生成高温热塑性的铝并 被挤出界面形成飞边, 扭矩逐渐降低到一个低谷, 暂且把低谷扭矩值称为稳态扭矩, 此时扭矩曲线并 没有持续一个平台; (5)对应顶锻阶段, 扭矩直接上 升, 这也意味着顶锻过程的开始, 随后到达后峰值 扭矩, 摩擦停止后经保压、冷却焊接过程结束。

\section{2 转速对焊接扭矩和能量输入的影响}

图 5 所示为摩擦压力 $30 \mathrm{MPa}$ 、顶锻压力 60 $\mathrm{MPa}$ 、摩擦缩短量 $5 \mathrm{~mm}$ 时不同转速条件下的接头 宏观形貌。转速为 $300 \mathrm{r} / \mathrm{min}$ 时由于焊接扭矩过大造 成主轴过载发生了 “闷车”, 这也使得铝侧母材发生 严重扭曲变形; 转速为 $500 \mathrm{r} / \mathrm{min}$ 时接头飞边未卷 起, 呈 “墩粗” 状; 转速达到 $800 \mathrm{r} / \mathrm{min}$ 时, 接头处 
形成了完整的飞边, 且飞边与铝母材之间的金属存 在明显的塑性变形; 转速达到 $1500 \mathrm{r} / \mathrm{min}$ 及以上时, 母材金属既无明显变形且形成的飞边也更加细淢。 以上低转速下发生失稳摩擦应该是接头高扭矩和接 头温度分布不均匀造成的, 低转速下接触时焊接扭 矩大, 界面附近的铝瞬时温度很高已达热塑性状态,
但这一过程发生速度快, 温度来不及传导至接头附 近的铝母材, 由于温度较低, 接头附近的铝母材依 然处于弹性状态，高扭矩使其发生扭曲变形。可以 看出, 接头铝侧母材既不发生扭曲, 又能形成均匀、 完整飞边的焊接临界转速在 $800 \mathrm{r} / \mathrm{min}$ 和 $1500 \mathrm{r} / \mathrm{min}$ 之间。

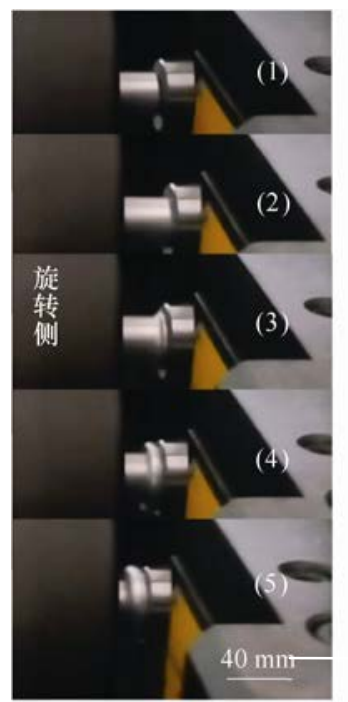

(a) 接头结合形貌

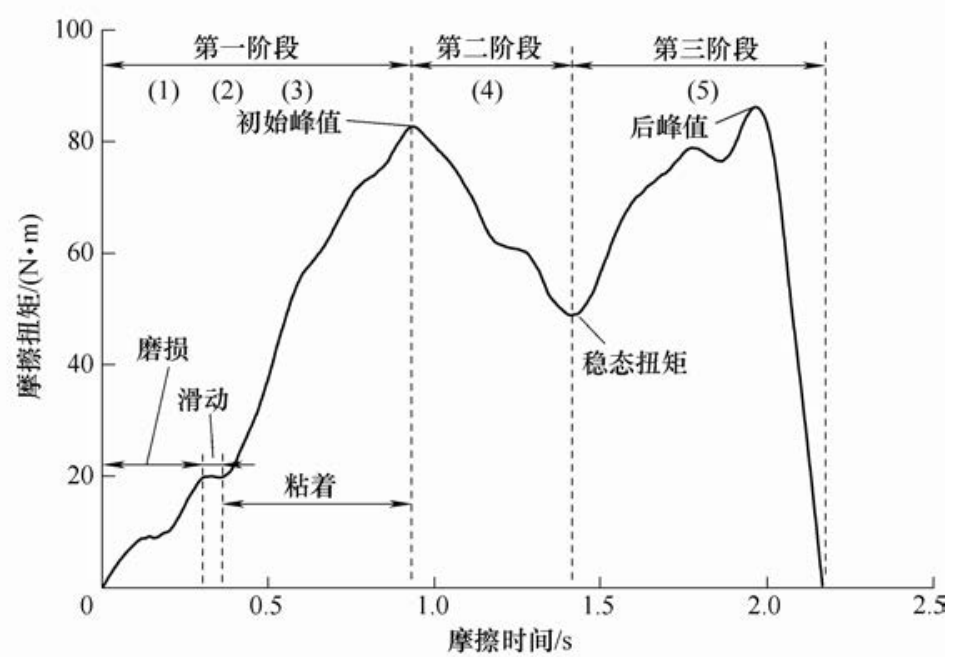

(b) 焊接接头摩擦扭矩变化曲线

图 4 焊接过程中接头的结合形貌和摩擦扭矩特征

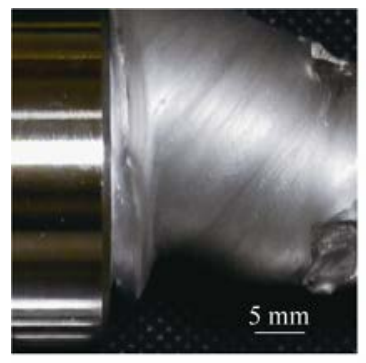

(a) $300 \mathrm{r} / \mathrm{min}$

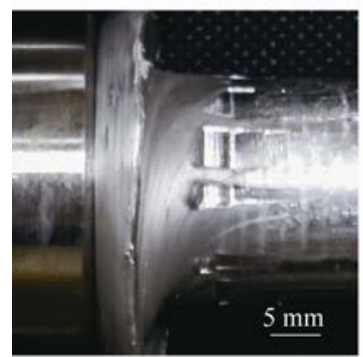

(c) $800 \mathrm{r} / \mathrm{min}$

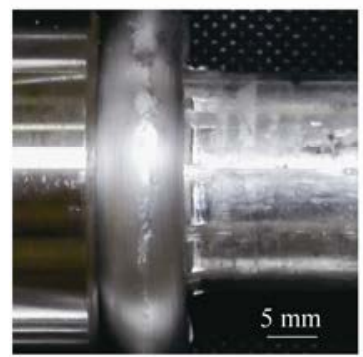

(e) $2000 \mathrm{r} / \mathrm{min}$

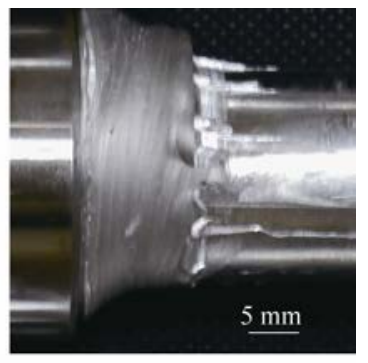

(b) $500 \mathrm{r} / \mathrm{min}$

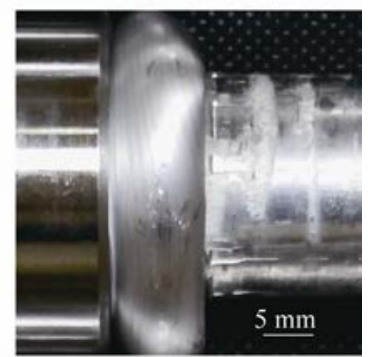

(d) $1500 \mathrm{r} / \mathrm{min}$

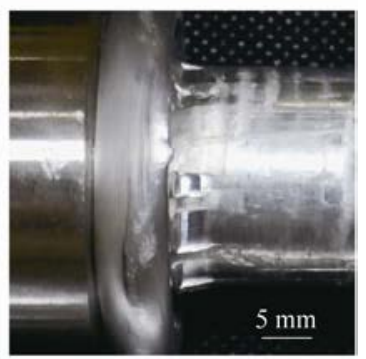

(f) $2500 \mathrm{r} / \mathrm{min}$
图 5 不同转速下接头的宏观形貌
不同转速条件下接头的摩擦扭矩变化曲线如图 6a 所示, 当转速低于 $1500 \mathrm{r} / \mathrm{min}$ 时, 扭矩曲线只存 在一个明显的峰值, 转速在 $1500 \mathrm{r} / \mathrm{min}$ 及以上时扭 矩曲线均呈现初始峰值和后峰值特征, 即随焊接过 程的进行扭矩先逐渐上升到初始峰值, 之后下降到 一个低谷, 然后随着顶锻的开始又上升到后峰值。 接头摩擦扭矩曲线存在明显前后峰值特征意味着焊 接接头外观成形良好, 若只存在一个峰值或两个峰 值不明显, 则有可能发生了失稳摩擦。图 6b 所示为 转速在 $1500 \mathrm{r} / \mathrm{min}$ 及以上时初始峰值扭矩、到达初 始峰值扭矩的时间、稳态扭矩、准稳态阶段持续时 间以及后峰值扭矩的变化规律。随着转速的升高, 初始峰值扭矩、稳态扭矩和后峰值扭矩随转速的升 高总体呈减小趋势, 初始峰值扭矩到达的时间有略 微波动, 但变化不太明显。准稳态阶段的持续时间 随转速的升高呈上升趋势, 这是由于准稳态扭矩随 转速升高而下降, 而摩擦缩短量都是一致的, 所以 就需延长摩擦时间来完成相同的摩擦缩短量。

图 7a 为不同转速条件下焊接过程第一阶段的 能量输入特征, 图 $7 \mathrm{~b}$ 为焊接各阶段及全过程的能量 输入特征。第一阶段是接头热量的积累阶段, 当焊 接开始时两端面接触发生磨损(Abrasion), 然后发生 滑动摩擦(Slide), 由图 7a 可以看出, 接头磨损和滑 动摩擦的产热量较小，通常这部分热量对接头起到 
了预热的作用。随着焊接过程的继续, 滑动摩擦转 为粘着摩擦(Stick), 接头热塑性状态的铝不断与钢 侧焊合后又被剪断, 粘着区域不断扩大, 接头产生 大量的热, 且粘着摩擦热对第一阶段产热的贡献占 主导作用。第一阶段产热占总产热的比率随转速的 升高基本维持不变; 接头全过程的能量输入随转速 的升高呈上升的趋势; 第二阶段的能量输入变化呈 现出与图 6 中该阶段的持续时间相同的变化趋势; 第三阶段的能量输入随转速的增加缓慢减小, 由于 转速和摩擦时间直接影响接头的温度和温度场, 第 一、二阶段的能量输入随转速的升高而升高, 这也 意味着顶锻开始前的温度是随转速的升高而升高 的, 此时接头摩擦因数可能会随转速的升高降低, 由式(6)可知顶锻阶段的摩擦加热功率也随转速的 升高而降低, 因此顶锻阶段的能量输入也会减小。

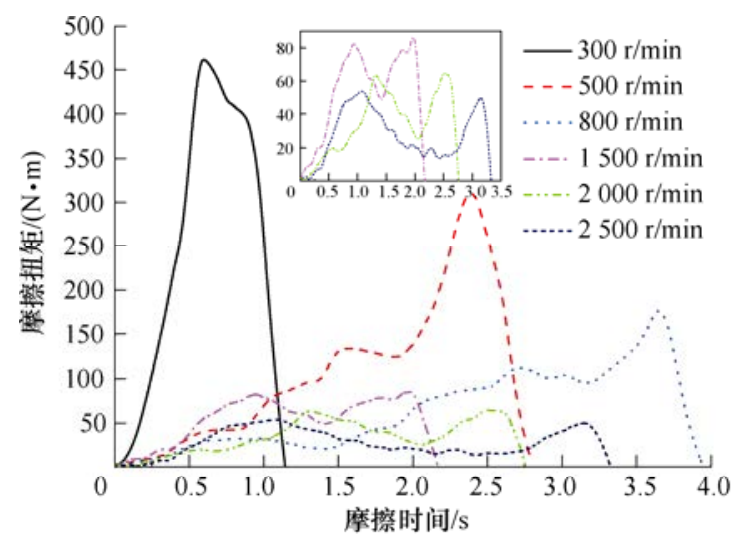

(a) 焊接全过程的摩擦扭矩

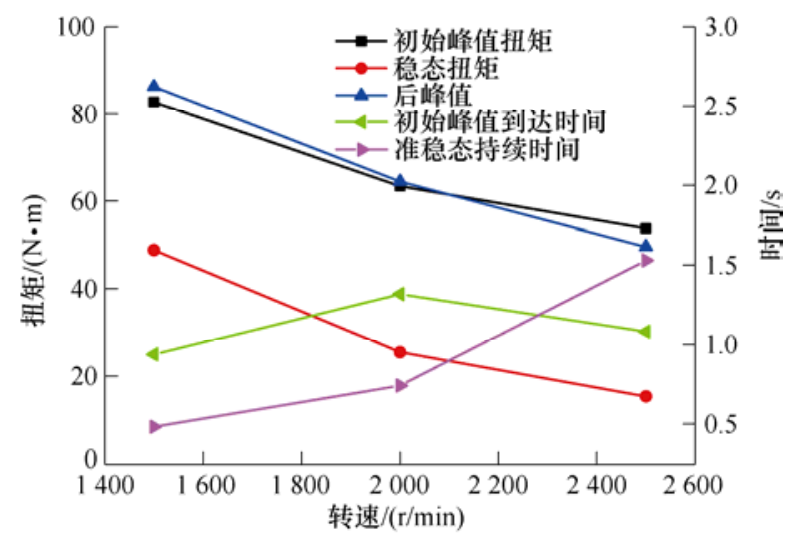

(b) 各特征扭矩的变化特征

图 6 不同转速下摩擦扭矩的特征

\section{3 顶锻压力对焊接扭矩和能量输入的影响}

图 8 为转速 $1500 \mathrm{r} / \mathrm{min}$ 、摩擦压力 $30 \mathrm{MPa}$ 、摩 擦缩短量 $5 \mathrm{~mm}$ 时不同顶锻压力条件下的接头宏观 形貌。顶锻压力为 $60 \mathrm{MPa} 、 80 \mathrm{MPa} 、 100 \mathrm{MPa}$ 下的 接头外观看不出明显区别, 但是当顶锻压力达到 $120 \mathrm{MPa}$ 时, 飞边明显增大, 这个压力已经远远超 过铝母材的屈服极限, 这就造成接头发生了严重“墩 粗”。通常顶锻压力的作用是挤出塑性层的高温金
属、氧化物和其他杂质, 并使焊缝得到锻造, 使其 晶粒细化，但顶锻压力过大则会使接头变形增大飞 边增多，浪费过多的母材金属同时留在接头界面的 塑性金属很少，严重时还会在焊缝中形成低温横向 流动的 “弯曲组织”, 使接头力学性能降低。

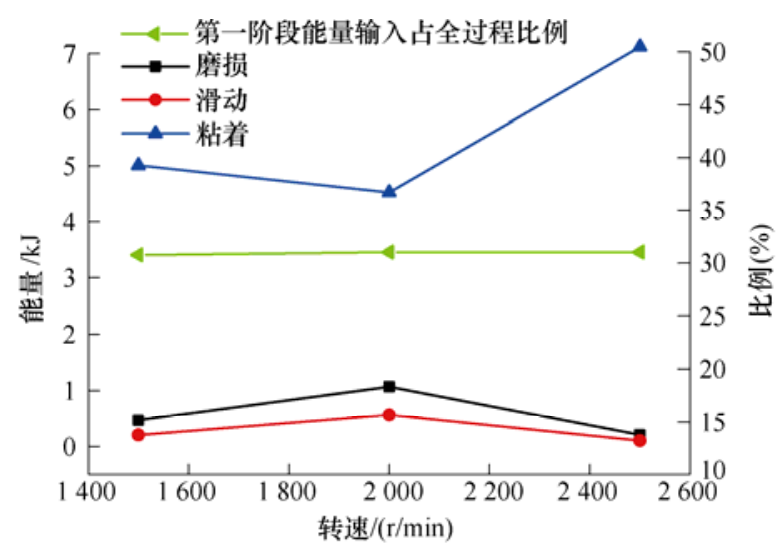

(a) 第一阶段能量输入特征

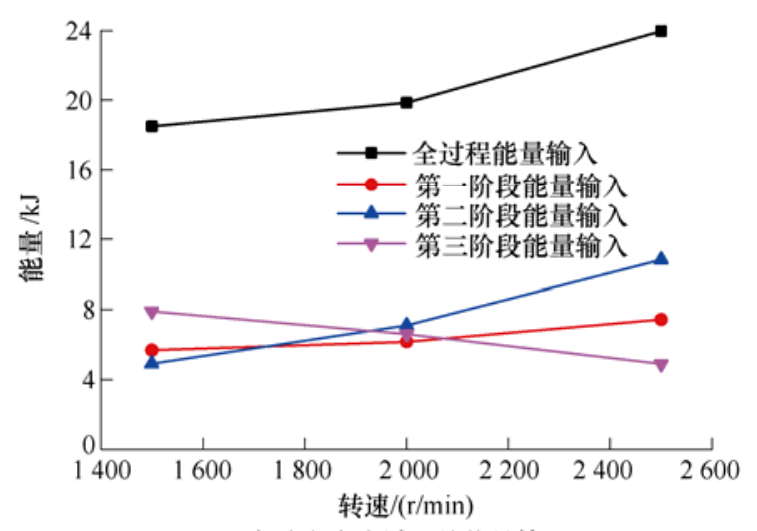

(b) 各阶段和全过程的能量输入

图 7 不同转速条件接头的能量输入特征

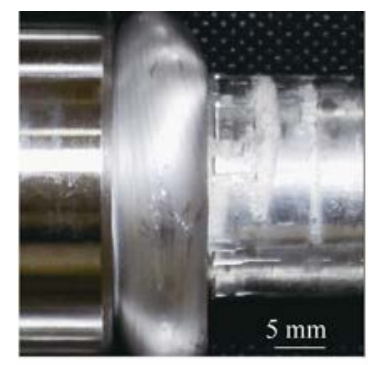

(a) $60 \mathrm{MPa}$

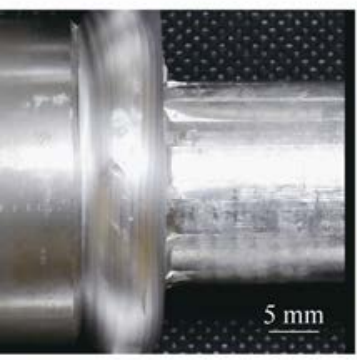

(c) $100 \mathrm{MPa}$

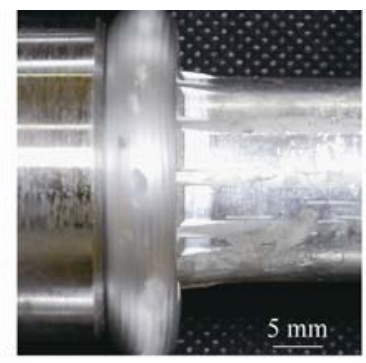

(b) $80 \mathrm{MPa}$

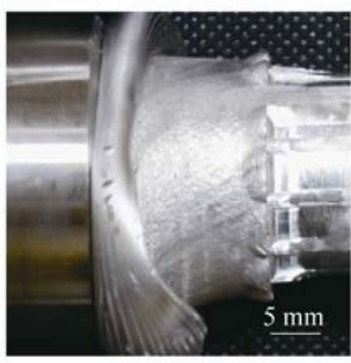

(d) $120 \mathrm{MPa}$
图 8 不同顶锻压力条件下的接头宏观形貌 
转速 $1500 \mathrm{r} / \mathrm{min}$ 、摩擦压力 $30 \mathrm{MPa}$ 、摩擦缩短 量 $5 \mathrm{~mm}$ 时不同顶锻压力条件下的摩擦扭矩变化曲 线如图 9a 所示, 扭矩曲线同样呈现初始峰值和后峰 值特征, 但由于转速和摩擦压力相同, 整个焊接过 程初始摩擦阶段扭矩的变化趋势基本一致, 初始峰 值扭矩有下降的趋势, 但是变化不大。随着顶锻压 力的增加, 后峰值扭矩也增加, 焊接总时间降低, 当顶锻压力达到 $80 \mathrm{MPa}$ 以后, 焊接总时间基本没 有变化。图 9b 所示为不同顶锻压力条件下后峰值扭 矩和到达后峰值扭矩的时间的变化趋势, 可以看出, 后峰值扭矩对顶锻压力的响应显著, 随着顶锻压力 的增加, 后峰值扭矩逐渐上升, 而到达后峰值扭矩 的时间的变化趋势则与之相反。

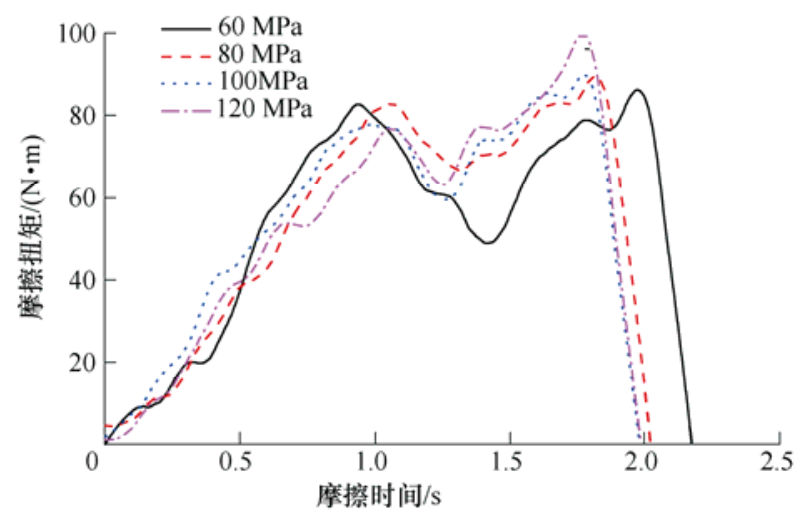

(a) 焊接全过程的摩擦扭矩

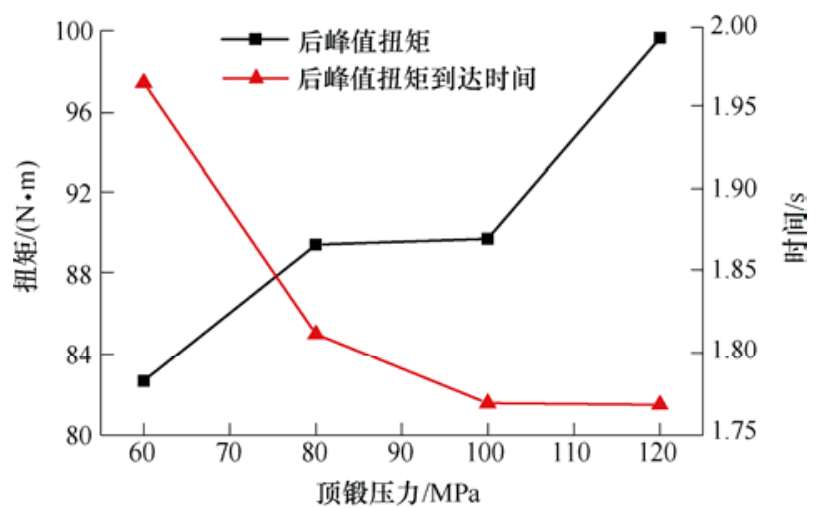

(b) 后峰值扭矩和到达后峰值扭矩时间的变化

图 9 不同顶锻压力下摩擦扭矩的特征

转速、摩擦压力、摩擦缩短量一定时, 顶锻压 力主要影响后峰值扭矩, 对第一阶段的加热过程影 响不大。图 10 所示为不同顶锻压力条件下第三阶段 的能量输入特征。随着顶锻压力的增加, 接头总能 量输入呈缓慢降低的趋势, 第三阶段能量输入的变 化则与之相反, 呈缓慢升高的趋势。图中的绿线为 第三阶段能量输入在总能量输入中的占比, 可以看 出其随顶锻压力的升高而逐渐增大, 且变化明显, 特别是当顶锻压力为 $120 \mathrm{MPa}$ 时, 第三阶段能量输 入占比高达 50\%, 这说明高顶锻压力条件下, 第三
阶段的能量输入对焊接接头的成形和焊后质量起到 了至关重要的作用。

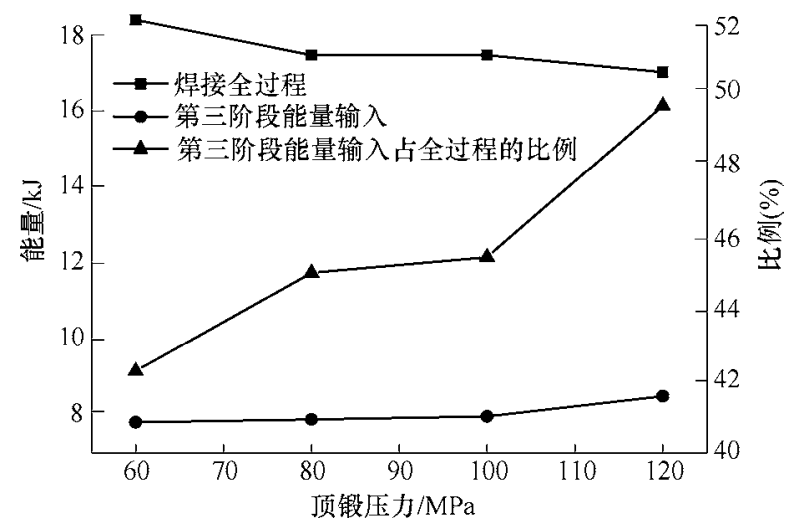

图 10 不同顶锻压力条件下第三阶段的能量输入特征

\section{3 结论}

(1) 根据摩擦扭矩的特征, 焊接过程可划分为 初始摩擦阶段、准稳态阶段和顶锻阶段, 初始摩擦 阶段又可分为磨损、滑动摩擦和粘着摩擦三个阶段, 滑动摩擦阶段对应于初始摩擦阶段中的扭矩平台; 初始摩擦阶段是接头热量的积累阶段, 这部分热量 以粘着摩擦产热为主。

(2) 转速的影响: 转速较低时, 摩擦扭矩曲线 只存在一个峰值或前后峰值特征不明显, 此时发生 失稳摩擦; 随着转速升高, 接头摩擦扭矩降低, 准 稳态阶段的持续时间则相反, 初始摩擦阶段、准稳 态阶段和焊接全过程的能量输入都增加, 但顶锻阶 段由于顶锻过程摩擦加热功率的降低而缓慢减小。

(3) 顶锻压力的影响: 焊接过程中后峰值扭矩 和顶锻压力呈正相关; 顶锻压力增加, 接头总能量 输入呈缓慢降低的趋势, 顶锻阶段能量输入变化与 之相反, 并且其在总能量输入中的占比逐渐增大, 这表明高顶锻压力下，顶锻阶段的能量输入对焊接 接头的成形和焊后质量起到了至关重要的作用。

\section{参 考 文 献}

[1] 中国机械工程学会焊接学会. 焊接手册 [M]. 北京: 机 械工业出版社, 1992.

Welding Society of Chinese Mechanical Engineering Society. Welding handbook[M]. Beijing: China Machine Press, 1992.

[2] KEARNS W H. Welding handbook: Resistance and solid-stata welding and other joining processes[M]. Miami: American Welding Society, 2007.

[3] 刘中青, 刘凯. 异种金属焊接技术指南[M]. 北京: 机 械工业出版社, 1997. 
LIU Zhongqing, LIU Kai. Manual of dissimilar metal welding technology[M]. Beijing: China Machine Press, 1997.

[4] LI W, VAIRIS A, PREUSS M, et al. Linear and rotary friction welding review[J]. International Materials Reviews, 2016, 61(2): 71-100.

[5] 傅莉. 电场对摩擦焊缝组织与性能的影响 [D]. 西安: 西 北工业大学, 2002.

FU Li. Influence of electric field on the Microstructures and mechanical properties of friction welding Joint[D]. Xi’an: Northwestern Polytechnical University, 2002.

[6] YILBAS B S, SAHIN A Z. Friction welding[M]. Berlin Heidelberg: Springer, 2014.

[7] 张雪超, 熊江涛, 李京龙, 等. 恒进给旋转摩擦条件下 金属力学参量周期响应行为 [J]. 机械工程学报, 2015, 51(2): 65-70.

ZHANG Xuechao, XIONG Jiangtao, LI jingling, et al. Periodic variation of torque and axial force of tubular aluminum alloy at constant downward and rotational speed[J]. Journal of Mechanical Engineering, 2015, 51(2): $65-70$.

[8] KIMURA M, KUSAKA M, SEO K, et al. Experimental study of seizure phenomena in weld interface of steel friction welds. Study of joining mechanism of friction welding (5report)[J]. Welding International, 2006, 20(4): 275-283.

[9] KIMURA M, INOUE H, KUSAKA M, et al. Analysis method of friction torque and weld interface temperature during friction process of steel friction welding[J]. Journal of Solid Mechanics \& Materials Engineering, 2010, 4(3): 401-413.

[10] LI P, LI J, LI X, et al. A study of the mechanisms involved in initial friction process of continuous drive friction welding[J]. Journal of Adhesion Science and Technology, 2015, 29(12): 1246-1257.
[11] 刘金合, 罗健. 连续驱动摩擦焊动态扭矩检测的新方法 —VCMM 法[J]. 西北工业大学学报, 1996(4): 649-650. LIU Jinhe, LUO Jian. On measuring dynamic torque acting on work piece in continuous drive friction welding[J]. Journal of Northwestern Polytechnical University, 1996(4): 649-650.

[12] LUO J. New measurement technique and model for friction torque in continuous drive friction welding: engineering oriented voltage-current floating evaluation measurement method and exponential function model of friction torque[J]. Science \& Technology of Welding \& Joining, 2015, 6(4): 209-212.

[13] 杜随更, 傅莉, 漂君辉, 等. 摩擦焊接过程电液比例计 算机闭环控制系统研制 [J]. 机械工程学报，2002, 38(12): 135-137.

DU Suigeng, FU Li, YAN Junhui, et al. On the computer closed loop control system with electron hydraulic proportional valve during friction welding[J]. Journal of Mechanical Engineering, 2002, 38(12): 135-137.

[14] 王笑川, 徐晓菱. 摩擦焊模块化测控系统实现[J]. 机械 工程学报, 2007, 43(2): 215-218.

WANG Xiaochuan , XU Xiaoling. Realization of modularized friction welding measure and control system[J]. Chinese Journal of Mechanical Engineering, 2007, 43(2): 215-218.

[15] MAALEKIAN M, KOZESCHNIK E, BRANTNER H P, et al. Comparative analysis of heat generation in friction welding of steel bars[J]. Acta Materialia, 2008, 56(12): 2843-2855.

作者简介: 张昌青, 男, 1974 年出生, 副研究员, 硕士研究生导师。主 要研究方向为先进材料的摩擦焊、钎焊及阻焊等固相连接的基础理论与 应用技术。

E-mail: zhangcq@lut.cn

刘雄波(通信作者), 男, 1992 年出生, 硕士研究生。主要研究方向为异 种金属的摩擦焊接。

E-mail: clipo@foxmail.com 